\title{
SOME TOPOLOGICAL PROPERTIES OF CONVEX SETS $\left({ }^{(}\right)$
}

\author{
BY \\ V. L. KLEE, JR.
}

Introduction. This paper is a sequel to [13] and the (more or less standard) notation of the latter is used without further explanation. However, explicit dependence is slight, and most of the present paper can be read independently of $[13]$.

Contents are indicated by section headings: $\S 1$. Compact convex sets. $\S 2$. Some spaces which lack the fixed-point property. \$3. Extension of homeomorphisms. §4. The mappings of Keller. §5. Topological representatives of convex sets.

In $\S 1$ it is proved that a compact convex subset of a normed linear space must be homeomorphic with a parallelotope, and this fact is used to show that all infinite-dimensional separable Banach spaces are of the same dimension-type. $\$ 2$ complements a well-known fixed-point theorem by showing that a convex subset of a normed linear space has the fixed-point property only if it is compact. $\$ 3$ shows that if $X$ is a compact subset of Hilbert space $\mathfrak{S}$ and $f$ is a homeomorphism of $X$ into $\mathfrak{S}$, then there is an isotopy $\eta$ of $\mathfrak{S}$ onto $\mathfrak{S}$ such that $\eta_{0}$ is the identity on $\mathfrak{S}$ and $\eta_{1} \mid X=f$. $\S 4$ elaborates a mappingtechnique introduced by Keller, and in particular strengthens the known homogeneity properties of the Hilbert parallelotope. $\$ 5$ shows that if $C$ is a locally compact closed convex subset of a normed linear space, then there are cardinal numbers $m$ and $n$ with $0 \leqq m \leqq \boldsymbol{\aleph}_{0}$ and $0 \leqq n<\boldsymbol{\aleph}_{0}$ such that $C$ is homeomorphic with either $\left.[0,1]^{m} \times\right] 0,1\left[^{n}\right.$ or $[0,1]^{m} \times[0,1[$.

Throughout the paper, $\Re$ will denote the real number field and $\mathfrak{P}$ the Hilbert parallelotope $\prod_{1}^{\infty}[-1 / n, 1 / n] \subset\left(l^{2}\right)$. By topological linear space we shall mean a vector space $E$ over $\Re$ with an associated Hausdorff topology in which the linear operations $x+y \mid(x, y) \in E \times E$ and $r x \mid(r, x) \in \Re \times E$ are continuous.

1. Compact convex sets. We extend here a theorem of Keller [11], which asserts that every infinite-dimensional compact convex subset of Hilbert space $\mathfrak{S}$ is homeomorphic with the Hilbert parallelotope $\mathfrak{B}$. The material of this section might more naturally appear as part of $\S 5$, but since it is much simpler than the latter, it is given here for easy accessibility.

If $A$ and $B$ are linear spaces and $X$ is a subset of $A$, then by a linear transformation of $X$ into $B$ we shall mean the restriction to $X$ of a linear transformation of $A$ into $B$. A basic tool is

Presented to the Society, June 19, 1954 ; received by the editors July 23, 1953.

(1) Sponsored in part by the Office of Ordnance Research, U. S. Army, under Contract DA-04-200-ORD-292. 
(1.1) Suppose $K$ is a compact convex subset of a topological linear space $E$, and there is a countable set $F$ of continuous linear functionals on $K$ such that for any two different points $x$ and $y$ of $K$ there is an $f \in F$ for which $f x \neq f y$. Then there is a linear homeomorphism of $K$ into Hilbert space $\mathfrak{W}$.

Proof. Let $F=\left\{f_{1}, f_{2}, \cdots\right\}$. For each $n$, $\sup _{K}\left|f_{n}\right|=B_{n}<\infty$; let $g_{n}$ $=\left(n B_{n}\right)^{-1} f_{n}$. Let $t$ be the original topology of $K$ and $t^{\prime}$ the weak topology generated by $\left\{g_{1}, g_{2}, \cdots\right\}$. Then $K_{t}$ is compact, $K_{t^{\prime}}$ is a Hausdorff space, and $t^{\prime} \subset t$, so $t^{\prime}=t$. For each $x \in K$, let $T x=\left(g_{1} x, g_{2} x, \cdots\right) \in \mathfrak{B} \subset\left(l^{2}\right)=\mathfrak{W}$. Clearly $T$ is a biunique continuous transformation of $K_{t}$ into $\mathfrak{F}$, and hence must be a homeomorphism of $K_{t}$ into $\mathfrak{S}$. It remains to show that $T$ is a linear transformation of $K$ into $\mathfrak{S}$.

For each $n$, there is a linear functional $h_{n}$ on $E$ such that $g_{n}=h_{n} \mid K$. Let $L$ be the linear hull of $K$ in $E$ and for each $x \in L$, let $T_{1} x=\left(h_{1} x, h_{2} x, \cdots\right)$ $\in\left(l^{2}\right)$. Then $T_{1}$ is a linear transformation of $L$ into $\mathfrak{S}$ such that $T_{1} \mid K=T$, and it is easy to extend $T_{1}$ to a linear transformation of $E$ into $\mathfrak{S}$. This completes the proof.

Combining (1.1) and Keller's theorem, we have

(1.2) Theorem. Suppose $S$ is a separable normed linear space, $E$ is either $S_{n}$ ( $S$ in its norm topology), $S_{w}$ ( $S$ in its weak topology), or $S_{w *}^{*}$ ( $S^{*}$ in its weak* topology), and $K$ is an infinite-dimensional compact convex subset of $E$. Then $K$ is homeomorphic with the Hilbert parallelotope.

(1.3) Corollary. Every infinite-dimensional compact convex subset of a normed linear space is homeomorphic with the Hilbert parallelotope.

The proof of (1.1) is similar to a proof of Arens [1, (4.3)]. The result (1.3) solves a problem proposed in $[13$, p. 35] and renders obsolete part of $\S I V 1$ of [13]. From (1.2) and an argument indicated in [13, p. 40], it follows that if $C$ is the unit cell, in the weak topology, of the complete inner-product space $\left(l^{2} n\right)$, then $K$ is homeomorphic with a parallelotope if and only if $n \leqq \boldsymbol{N}_{0}$.

In the terminology of Fréchet [7], two topological spaces are of the same dimension-type provided each is homeomorphic with a subset of the other. We note here

(1.4) CoRollary. All infinite-dimensional separable Banach spaces are of the same dimension-type.

Proof. As is well known, every separable metric space is homeomorphic with a subset of $\mathfrak{B}$, so it suffices to show that an arbitrary infinite-dimensional Banach space $E$ must contain a homeomorph of $\mathfrak{P}$. Let $x_{\alpha}$ be a linearly independent sequence of elements of $E$, with $x_{\alpha} \rightarrow \phi$, and let $C$ be the closed convex hull of $\left\{\phi, x_{1}, x_{2}, \cdots\right\}$. By a theorem of Mazur ([17] and [3, p. 81]), $C$ is compact, and hence by (1.3) is homeomorphic with $\mathfrak{B}$. This completes the proof. 
A much more difficult question remains open: Are all infinite-dimensional separable Banach spaces mutually homeomorphic? (See [2, p. 242] and [7, pp. 95 and 143 ].)

It is well knowr $[2$, p. 185] that every separable Banach space is equivalent to a complete linear space of continuous real functions on $[0,1]$, in the usual "sup" norm. In closing this section, we note an alternative representation, as follows:

(1.5) If $E$ is a separable Banach space, there is a compact symmetric convex subset $C$ of Hilbert space such that $E$ is equivalent to $L_{C}$, the space of all bounded linear functionals on $C$, in the "sup" norm.

Proof. Let $Q$ be the unit cell of $E^{*}$. By (1.1) there is a linear homeomorphism $T$ of $Q_{w^{*}}$ into $\mathfrak{S}$. Let $C=T Q$ and for each $x \in E$ let $u x=u_{x} \in L_{C}$, where $u_{x} p=\left(T^{-1} p\right) x$ for each $p \in C$. Then $u$ is a linear isometry of $E$ onto $L_{C}$.

2. Some spaces which lack the fixed-point property. A topological space $S$ is said to have the fixed-point property iff every continuous map of $S$ into $S$ leaves at least one point invariant. A well-known theorem of Tychonoff [21] asserts:

(a) If $E$ is a locally convex topological linear space and $K$ is a compact convex subset of $E$, then $K$ has the fixed-point property.

We obtain here a partial converse, as follows:

(b) If $E$ is a locally convex metrizable topological linear space and $K$ is a noncompact convex subset of $E$, then $K$ lacks the fixed-point property.

It is apparently unknown whether either (a) or (b) is valid in an arbitrary topological linear space. In the case of (a), in particular, this is an important unsolved problem.

By topological ray is meant a homeomorphic image of the half-open interval $[0,1[$. Our fundamental tool in showing that certain spaces lack the fixed-point property is

(2.1) If $S$ is a normal space which contains a topological ray $T$ as a closed subset, then there is a null-homotopic map without fixed point of $S$ into $S$.

Proof. Clearly there is a null-homotopic map $m$ without fixed point of $T$ into $T$. By a slight addition to the Tietze-Urysohn extension theorem, there is a retraction $r$ of $S$ onto $T$. Then $m r$ is the desired map of $S$ into $S$.

We wish next to show that a noncompact convex subset $K$ of a topological linear space $E$ must contain a topological ray as a relatively closed subset, but are actually able to do this only under various additional hypotheses. Even if it could be proved without further assumptions, our method would establish the result (b) for $E$ only under the additional assumption that $K$ is normal (automatically true if $E$ is metrizable).

A subset $B$ of a topological linear space is bounded iff for each open $U \ni \phi$ there is a number $t$ such that $t U \supset B$. A set is linearly bounded iff its inter- 
section with each line is bounded. A topological linear space is locally 〈linearly bounded iff it contains a nonempty 〈linearly〉 bounded open set.

(2.2) Suppose $E$ is a topological linear space and $C$ is a convex subset of $E$. Then if at least one of the following statements is true, $C$ must contain a topological ray as a relatively closed subset:

(i) $E$ is locally convex and $C$ is unbounded;

(ii) $E$ is metrizable and $C$ is not complete in the natural uniformity;

(iii) $E$ is isomorphic with a subspace of a product of locally linearly bounded topological linear spaces, and some bounded subset of $C$ fails to be precompact;

(iv) $C$ is closed, locally compact, and unbounded;

(v) $E$ is locally convex and metrizable, $C$ is not compact;

(vi) $E$ is locally bounded, $C$ is not compact.

Proof. As a first step in proving (2.2), we note the sufficiency of the condition (0) $C$ is finite-dimensional and noncompact. For in this case $C$ either fails to be linearly bounded or fails to be linearly closed, and the desired conclusion follows at once.

(i) By a theorem of Mackey [16, p. 524], there is a continuous linear functional $f$ on $E$ such that $\sup _{c} f=\infty$. Let $p_{0} \in C$ and for each positive integer $n$ choose $p_{n} \in C$ such that $f p_{n}=f p_{0}+n$. Let $T=\cup_{1}^{\infty}\left[p_{i}, p_{i+1}\right]$. Then $T$ is a closed subset of $C$ and is mapped homeomorphically by $f$ onto the ray $\left[f p_{1}, \infty[\right.$.

(ii) We may suppose that $E$ is contained in its completion $E^{\prime}$ and that (relative to $E^{\prime}$ ) $q \in \mathrm{Cl} C \sim C$. In view of (0), we may assume that $C$ is infinitedimensional. Let $U_{\alpha}$ be a fundamental sequence of neighborhoods of $q$, and choose $p_{1} \in U_{1} \cap C$. Then the choice of $p_{2}, p_{3}, \cdots$ is carried out inductively as follows: Having chosen $p_{1}, \cdots, p_{k}$, let $L_{k}$ be the linear hull of $\left\{p_{1}, \cdots, p_{k}\right\}$. Since $C$ is infinite-dimensional and $q \in \mathrm{Cl} C \sim C$, it follows easily that $C \cap U_{k+1}$ is not contained in $L_{k}$; choose the point $p_{k+1}$ from $C \cap U_{k+1} \sim L_{k}$. Now with $T=\cup_{1}^{\infty}\left[p_{i}, p_{i+1}\right], T$ does not "cross itself" and can be seen to be the desired topological ray.

(iii) By the condition on $C$, there are a bounded infinite subset $X$ of $C$ and a neighborhood $U$ of $\phi$ in $E$ such that $x-x^{\prime} \notin U$ whenever $x$ and $x^{\prime}$ are different points of $X$. By the condition on $E$, we may assume that there are topological linear spaces $A$ and $B$ and a linearly bounded subset $Q$ of $B$ for which $E \subset A \times B$ and $(A \times Q) \cap E$ is a closed neighborhood $V$ of $\phi$ in $E$ such that $[-1,1] V=V$ and $V+V+V+V \subset U$. Since $X$ is bounded, there are a symmetric neighborhood $W$ of $\phi$ in $E$ and an integer $m$ such that $W \subset V$ and $X+W \subset m V$. We shall establish the existence of a sequence $p_{\alpha}$ of points of $X$ such that with $L_{k}$ denoting the linear hull of $\left\{p_{1}, \cdots, p_{k}\right\}$ it is always true that $p_{k+1}+W$ misses $L_{k}$. It is then easy to see that $U_{1}^{\infty}\left[p_{i}, p_{i+1}\right]$ is the desired topological ray.

To guarantee the existence of the sequence $p_{\alpha}$ as described, it clearly suffices to show that if $L$ is an arbitrary finite-dimensional linear subspace of 
$E$, then $x+W$ misses $L$ for some $x \in X$. Let $V^{\prime}=L \cap V$ and $A^{\prime}=L \cap(A \times\{\phi\})$. Let $M$ be a subspace complementary to $A^{\prime}$ in $L$ and let $J=M \cap V^{\prime}$. It can be verified that $V^{\prime}=A^{\prime}+J$. Now $J$ is a closed neighborhood of $\phi$ in $M$ and $J$ is linearly bounded and star-shaped from $\phi$. It then follows, since $M$ is finitedimensional, that $J$ is bounded and precompact. Thus $m J$ is precompact, and since $V^{\prime}=A^{\prime}+J$ it follows that there is a finite subset $Y$ of $m J$ such that $m V^{\prime} \subset U_{\nu \in Y}\left(y+V^{\prime}\right)$. Now if $x \in X$ and $x+W$ intersects $L$, then $x+W$ intersects $(X+W) \cap L \subset(m V) \cap L=m V^{\prime}$, and hence $x+W$ intersects $y+V^{\prime}$ for some $y \in Y$. But if $x+W$ and $x^{\prime}+W$ both intersect $y+V$, then (since $V$ and $W$ are symmetric and $W \subset V) x-x^{\prime} \in V+V+V+V \subset U$, an impossibility if $x$ and $x^{\prime}$ are different points of $X$. Since $X$ is infinite and $Y$ finite, it follows that $x+W$ misses $L$ for some $x \in X$, and the proof is complete for case (iii).

(iv) We may suppose $\phi \in C$. Since $C$ is unbounded, there is an open set $U \ni \phi$ with $U=[-1,1] U$ such that for each $i, C \nsubseteq i U$. Since $C$ is locally compact, there is a subset $V$ of $E$ such that $\phi \in \operatorname{Int} V \subset V \subset U$ and $V \cap C$ is compact. Let $F$ be the boundary of $V$ and $Q=F \cap C$. Then $Q$ is compact, $\phi \notin Q$, and $C \subset\left[0, \infty\left[Q\right.\right.$. For each $i$ there are a point $q_{i} \in Q$ and a number $\left.t_{i} \in\right] 0, \infty[$ such that $t_{i} q_{i} \in C \sim i U$. Suppose $t_{\alpha}$ does not approach $\infty$. Then some subsequence of $t_{\alpha}$ converges to a number $t \in[0, \infty[$. Since $Q$ is compact, the corresponding subsequence of $q_{\alpha}$ has a cluster point $q \in Q$ and we have $t q \in C \sim i U$ for each $i$, an impossibility. Thus $t_{\alpha} \rightarrow \infty$, and since $C$ is closed and convex and $\phi \in C$ we have $\left[0, \infty\left[y \subset C\right.\right.$, where $y$ is an arbitrary cluster point of $q_{\alpha}$. The proof is complete for case (iv).

(v) If $C$ is unbounded or noncomplete, (i) or (ii) implies the desired conclusion. In the remaining case, $C$ is bounded and complete but not compact. Since a complete precompact space must be compact, it follows that $C$ is not precompact. And since every locally convex topological linear space is isomorphic with a subspace of a product of normed linear spaces [3, p. 99], (iii) now applies to give the desired conclusion.

(vi) The only case not covered by either (ii) or (iii) is that in which $C$ is complete and every bounded subset of $C$ is precompact. This case is covered by (iv).

The proof of (2.2) has now been completed. Combining (2.2) (v), (2.1), and Tychonoff's theorem, we have

(2.3) Theorem. For a convex subset $K$ of a locally convex metrizable topological linear space $E$, the following assertions are equivalent: $(\alpha) K$ is compact; $(\beta) K$ has the fixed-point property; $(\gamma)$ no relatively closed subset of $K$ is a topological ray.

We remark that (2.2) (iii) (and hence (2.3)) is more easily handled in the case of a normed linear space. It would be interesting to determine whether every topological linear space is isomorphic with a subspace of a product of locally $\langle$ linearly $\rangle$ bounded topological linear spaces. It is easy to see that every 
topological linear space is isomorphic with a subspace of a product of metrizable topological linear spaces.

(2.3) and its proof are analogous to work of Dugundji [5]. He showed that if $C$ and $S$ are respectively the unit cell and the unit sphere of an infinite-dimensional normed linear space, then $C$ can be retracted onto $S$, whence $C$ must lack the fixed-point property. Kakutani [10] and the author [13] showed that in a large class of infinite-dimensional normed linear spaces, the unit cell actually admits a homeomorphism onto itself without fixed point. By (5.8) (given later in this paper), it follows that such a homeomorphism is admitted also by every convex set $C$ in a normed linear space such that $C$ is noncompact, closed, locally compact, and at least two-dimensional.

We conclude this section by showing that certain other familiar spaces lack the fixed-point property. (2.4) and (2.5) below are obvious.

(2.4) If $S$ is a topological space which has the fixed-point property, then so has every retract of $S$.

(2.5) If $S$ has the fixed-point property, then $S$ is connected.

(2.6) Suppose $S$ is a noncompact, connected, locally connected, locally compact metric space. Then $S$ contains a topological ray as a closed subset.

Proof. By a result of Sierpinski [18], $S$ must be separable. Let $Y$ be the one-point compactification of $S$, with $Y=S \cup\{p\}$. $Y$ admits a countable basis for open sets and hence is metrizable. If $Y$ is not locally connected it contains a nondegenerate continuum at each point of which it fails to be locally connected [22, p. 19]. Since this contradicts the fact that $S$ is locally connected, $Y$ must be locally connected and hence arcwise connected [22, p. 36]. For an arbitrary $\operatorname{arc} A$ in $Y$ having $p$ as an end point, $A \cap S$ is a topological ray closed in $S$.

From (2.1) and (2.4)-(2.6) we have

(2.7) Theorem. If $S$ is a noncompact, locally connected, locally compact metric space, then $S$ lacks the fixed-point property.

From (2.1), (2.6), and known properties of ANR's [15], we have

(2.8) Suppose $X$ is a locally compact connected $A N R$. Then $X$ is compact if and only if every null-homotopic map of $X$ into $X$ leaves at least one point invariant.

An example: Let $Y$ be the set of all points $y=\left(y^{1}, y^{2}, \cdots\right)$ of the Hilbert space $\left(l^{2}\right)$ such that $\|y\| \leqq 1$ and $y^{i}$ is nonzero for at most one $i$. Then $Y$ is an $\mathrm{AR}$ and is locally compact at all but one point. However, $Y$ is noncompact and has the fixed-point property.

3. Extension of homeomorphisms. It was proved in [13] that if $E$ is either Hilbert space $\mathfrak{S}$ or a nonreflexive normed linear space and $Y$ is a compact subset of $E$, then there is an isotopy $\eta$ of $E$ into $E$ such that $\eta_{0}$ is the identity map on $E, \eta_{t} E=E$ for each $t \in\left[0,1\right.$ [, and $\eta_{1} E=E \sim Y$. Thus if $X$ 
and $Y$ are arbitrary compact subsets of $E, E \sim X$ is homeomorphic with $E \sim Y$. It is conceivable, nevertheless, that there exist such $X$ and $Y$, mutually homeomorphic, for which there is no homeomorphism simultaneously mapping $E$ onto $E$ and $X$ onto $Y$. We shall show that (at least in $\mathfrak{S}$ ) no such "wild" embedding is possible. The proof is based on three lemmas, of which the first is

(3.1) If $n$ is a non-negative integer and $Y$ is a compact subset of the infinitedimensional Banach space $E$, then there exist complementary closed linear subspaces $L_{1}$ and $L_{2}$ of $E$ and a homeomorphism $h$ of $E$ onto $E$ such that $h Y \subset L_{1}$ and $\operatorname{dim} L_{2}=n$.

Proof. For $n=0$, this is obvious. Suppose it has been proved for $n=k-1$ and consider the case $n=k$. By the inductive hypothesis, there are complementary closed linear subspaces $M_{1}$ and $M_{2}$ of $E$ and a homeomorphism $f$ of $E$ onto $E$ such that $f Y \subset M_{1}$ and $\operatorname{dim} M_{2}=k-1$. Each element $x$ of $E$ has a unique expression in the form $x=x_{1}+x_{2}$ with $x_{i} \in M_{i}$. By (II 3.1) of [13] there are complementary closed linear subspaces $L_{1}$ and $S$ of $M_{1}$ and a homeomorphism $g$ of $M_{1}$ onto $M_{1}$ such that $g f Y \subset L_{1}$ and $\operatorname{dim} S=1$. Let $L_{2}=M_{2}+S$, and let $h x=g(f x)_{1}+(f x)_{2}$ for each $x \in E$. It can be verified that $L_{1}, L_{2}$, and $h$ have the desired properties.

(3.2) If $Y$ is a compact subset of $\mathfrak{S}$, there exist complementary infinitedimensional closed linear subspaces $L_{1}$ and $L_{2}$ of $\mathfrak{S}$ and a homeomorphism $h$ of $\mathfrak{S}$ onto $\mathfrak{S}$ such that $h Y \subset L_{1}$.

Proof. By (II 3.1) of [13] there is a continuous linear functional $f$ on $\mathfrak{S}$ and a homeomorphism $g_{1}$ of $\mathfrak{F}$ onto $\mathfrak{S}$ such that $g_{1} Y$ is contained in the hyperplane $[f ; 1]$. Obviously, there is a homeomorphism $g_{2}$ of $\mathfrak{S}$ onto the open half-space $[f ;>0]$ such that $g_{2}$ is the identity map on $[f ; \geqq 1]$. Let $Z$ be the closed convex hull of $g_{1} Y$. By a theorem of Mazur [17], $Z$ is compact. By (II 3.3) there is a translate $Z^{\prime}$ of $Z$ with $Z^{\prime} \subset[f ; 0]$ and a homeomorphism $g_{3}$ of $[f ;>0]$ onto $[f ;>0] \cup Z^{\prime}$ such that $g_{3} Z=Z^{\prime}$. By the argument of (III 1.2) there are complementary infinite-dimensional closed linear subspaces $M_{1}$ and $L_{2}$ of $[f ; 0]$ and a homeomorphism $g_{4}$ of $[f ; \geqq 0]$ onto $[f ;>0]$ $\cup M_{1}$ which maps $[f ; 0]$ linearly onto $M_{1}$. Since $g_{4} Z^{\prime}$ is convex, there is by (II 3.3) a homeomorphism $g_{5}$ of $[f ;>0] \cup g_{4} Z^{\prime}$ onto $[f ;>0]$ which merely translates $g_{4} Z^{\prime}$ into $[f ; 1]$. Let $p \in g_{6} g_{4} Z^{\prime}, L_{1}=M_{1}+\{t p \mid-\infty<t<\infty\}$, and $h$ $=g_{2}^{-1} g_{5} g_{4} g_{3} g_{2} g_{1}$. It can be verified that $L_{1}, L_{2}$, and $h$ have the desired properties.

(3.3) Suppose $L_{1}$ and $L_{2}$ are complementary closed linear subspaces of the normed linear space $E, X$ is a closed subset of $L_{1}$ and $k$ is a homeomorphism of $X$ onto a closed subset of $L_{2}$. Then there is an isotopy $\zeta$ of $E$ onto $E$ such $\zeta_{0}$ is the identity map on $E$ and $\zeta_{1} \mid X=k$.

Proof. By a theorem of Dugundji [5], there are continuous maps $\alpha$ of $L_{1}$ into $L_{2}$ and $\beta$ of $L_{2}$ into $L_{1}$ such that $\alpha \mid X=k$ and $\beta \mid k X=k^{-1}$. Now for $x \in E$ and $t \in[0,1]$, let $\xi_{t} x=x+t\left(\alpha x_{1}\right)$ and $\eta_{t} x=x-t\left(\beta x_{2}\right)$, where $x=x_{1}+x_{2}$ with $x_{i} \in L_{i}$. It can be verified that $\xi$ and $\eta$ are isotopies of $E$ onto $E$. Now let 
$\zeta_{t}=\xi_{2 t}$ for $t \in[0,1 / 2]$ and $\zeta_{t}=\eta_{2 t-1} \xi_{1}$ for $t \in[1 / 2,1]$. Then if $x \in X$,

$$
\zeta_{1} x=\eta_{1} \xi_{1} x_{1}=\eta_{1}\left(x_{1}+\alpha x_{1}\right)=x_{1}+\alpha x_{1}-\beta \alpha x_{1}=x_{1}-k^{-1} k x_{1}+\alpha x=k x,
$$

so $\zeta$ is the desired isotopy.

(3.4) Theorem. Suppose $E$ is an infinite-dimensional Banach space, $X$ is a compact subset of $E$, and $f$ is a homeomorphism of $X$ into $E$. Suppose that either $E$ is Hilbert space or $X$ is finite-dimensional (or both). Then there is an isotopy $\mu$ of $E$ onto $E$ such that $\mu_{0}$ is the identity map on $E$ and $\mu_{1} \mid X=f$.

Proof. Let $Y=X \cup f X$. From (3.1) with $n=2 \operatorname{dim} X+1$ when $X$ is finitedimensional, and from (3.2) when $E=\mathfrak{S}$, we see that there exist complementary closed linear subspaces $L_{1}$ and $L_{2}$ of $E$, a homeomorphism $h$ of $E$ onto $E$ with $h Y \subset L_{1}$, and a homeomorphism $k$ of $h X$ into $L_{2}$. On $k h X$, let $k^{\prime}=h f h^{-1} k^{-1}$. Then $k^{\prime}$ is a homeomorphism of $k h X$ onto $h f X$. By (3.3) there are isotopies $\xi$ and $\eta$ of $E$ onto $E$ such that $\xi_{0}$ and $\eta_{0}$ are identity maps, $\xi_{1} \mid h X$ $=k$, and $\eta_{1} \mid k h X=k^{\prime}$. Let $\zeta_{t}=\xi_{2 t}$ for $t \in[0,1 / 2]$ and $\zeta_{t}=\eta_{2 t-1} \xi_{1}$ for $t \in[1 / 2,1]$. Then $\zeta$ is an isotopy of $E$ onto $E$ such that $\zeta_{0}$ is the identity map and $\zeta_{1} \mid h X$ $=h f h^{-1}$. Finally, let $\mu_{t}=h^{-1} \zeta_{t} h$ for each $t \in[0,1]$, and $\mu$ has the desired properties.

In closing this section, we apply (3.4) to obtain another extension theorem. This should be compared with results of Hausdorff $[8 ; 1$, p. 16] and Fox [6].

(3.5) Theorem. Suppose $E$ is an infinite-dimensional Banach space, $M$ is a separable metric space, $A$ is a compact subset of $M$, and $g$ is a continuous map of $A$ into $E$. Suppose that either $E$ is Hilbert space or $g A$ is finite-dimensional. Then there is a continuous map $g^{*}$ of $M$ into $E$ such that $g^{*} \mid A=f$ and $g^{*} \mid M \sim A$ is a homeomorphism. If $g$ is a homeomorphism, it can be arranged that $g^{*}$ is also a homeomorphism.

Proof. Let $M^{\prime}=\left\{g^{-1} y \mid y \in g A\right\} \cup\{\{x\} \mid x \in M \sim A\}$, metrized by the Hausdorff metric, and let $m$ be the natural map of $M$ into $M^{\prime}$. Then $M^{\prime}$ is a separable metric space, $m$ is continuous, $m$ is a homeomorphism if $g$ is, and there is a homeomorphism $k$ of $g A$ into $M^{\prime}$ such that $m \mid A=k g$. By (1.4) and the fact that every separable metric space is homeomorphic with a subset of $\mathfrak{S}$, there is a homeomorphism $h$ of $M^{\prime}$ into $E$. Let $f=k^{-1} h^{-1}\left|h m A=g m^{-1} h^{-1}\right| h m A$. Then $h m A$ is a compact subset of $E$ and $f$ is a homeomorphism of $h m A$ into $E$. By (3.4), there is a homeomorphism $f^{*}$ of $E$ onto $E$ such that $f^{*} \mid h m A=f$. With $g^{*}=f^{*} h m$, it can be seen that $g^{*}$ is the desired extension of $g$.

By use of an embedding theorem of Dowker [4] and the fact that every metric space is paracompact [20], the conclusion of (3.5) can be obtained also under the assumption that $E$ is a hyper-Hilbert space and $M$ is a metric space whose density character is not greater than that of $E$.

4. The mappings of Keller. If $S$ is a topological space and $n$ is a positive 
integer, then $S$ is $n$-point homogeneous iff for each pair $\left(x_{1}, \cdots, x_{n}\right)$ and $\left(y_{1}, \cdots y_{n}\right)$ of ordered $n$-tuples of distinct points of $S$, there exists a homeomorphism $h$ of $S$ onto $S$ such that $h x_{i}=y_{i}$ for $1 \leqq i \leqq n$. Keller [11] proved that the infinite-dimensional compact convex subsets of Hilbert space $\mathfrak{S}$ are all mutually homeomorphic and that each is one-point homogeneous. We shall here review and extend the method of Keller, in order to obtain certain refinements needed in $\S 5$, and to show that the infinite-dimensional compact convex sets are $n$-point homogeneous.

If $X$ is a convex set in a normed linear space and $x \in X$, then $x$ is a nonsupport point of $X$ iff there is no hyperplane which bounds $X$ and includes $x$. $X$ is elliptically convex iff the segment ] $p, q$ [ consists entirely of nonsupport points of $X$ whenever $X \ni p \neq q \in X$. It is important to note: (A) If $X$ is a complete separable convex set which is not contained in any hyperplane, then $X$ includes a nonsupport point. (B) If a convex subset of a strictly convexifiable space includes a nonsupport point, then it has an elliptically convex homeomorph. (For compact convex subsets of $\mathfrak{S}$, these results are both due to Keller. For the more general setting described above, the author claimed in [12] to prove (A), but the proof is valid only for compact $X$. However, Professor Ernest Michael has remarked that if $\phi \in X,\left\{x_{1}, x_{2}, \cdots\right\}$ is a dense subset of $X$, and $t_{\beta}=\min \left[\left\|x_{\beta}\right\|^{-1}, 1\right]$, then-assuming that $X$ is convex, complete, and not contained in any hyperplane- $-\sum_{1}^{\infty} 2^{-n} t_{n} x_{n}$ is a nonsupport point of $X$. The result (B) is proved in [13, (IV 1.2)] for bounded convex sets, and without this restriction in $\$ 5$ of the present paper.)

The arguments used by Keller in Hilbert space were extended in [13] to spaces with bases, and can in fact be applied in an arbitrary normed linear space. However, for present purposes it is more convenient to work exclusively in $\mathfrak{S}=\left(l^{2}\right)$, and then to make the transition to more general spaces by the methods of $\S 1$ and $\S 5$.

For each $x=\left(x^{1}, x^{2}, \cdots\right) \in \mathfrak{S}=\left(l^{2}\right)$ and non-negative integer $n, P_{n} x$ is defined by: $\left(P_{n} x\right)^{i}=x^{i}$ for $i \leqq n$ and $\left(P_{n} x\right)^{i}=0$ for $i>n$. For $X \subset \mathfrak{S}, X_{*_{n}} \equiv P_{n} X$.

Now suppose $K^{\prime}$ resp. $K$ is a compact elliptically convex subset of $\mathfrak{S}_{* r}$ resp. $\mathfrak{S C}_{* s}$, with $r \geqq s$. Suppose $0 \leqq t \leqq s$ and $g$ is a homeomorphism of $K_{* t}^{\prime}$ onto $K_{* t}$ (both of which are topological $t$-cells). By extending slightly the method of Keller, we shall define a homeomorphism $f=f_{K^{\prime}, g, K}$ of $K_{* s}^{\prime}$ onto $K$ such that $P_{t} f=g P_{t}$ on $K_{* s}^{\prime}$. (We permit here $s=\infty$, with $\mathfrak{S}_{*_{\infty}} \equiv \mathfrak{S}$, etc.)

For $x \in K$ and integer $n \geqq 0$, let $Q_{n} x=\left\{y \mid y \in K\right.$ and $\left.y_{*_{n}}=x_{*_{n}}\right\}$. Let $L_{n}$ resp. $H_{n}=\left\{x \mid x \in K\right.$ and $x^{n}=\min$ resp. $\left.\max \left\{y^{n} \mid y \in Q_{n-1} x\right\}\right\}$, and $E_{n}=L_{n}$ $\cup H_{n} . Q_{n}^{\prime} x^{\prime}$ (for $x^{\prime} \in K^{\prime}$ ), $H^{\prime} L^{\prime}$, and $E^{\prime}$ are defined similarly. Keller proves: (1) For each $x \in K$, the set $Q_{\beta-1} x \cap L_{\beta}$ resp. $Q_{\beta-1} x \cap H_{\beta}$ contains exactly one point (which we denote by $l_{\beta} x$ resp. $h_{\beta} x$ ). (2) $E_{\beta}=L_{\beta+1} \cap H_{\beta+1}$.

We shall define a set of mappings $\left\{f_{i} \mid t \leqq i<s+1\right\}$ such that $f_{i}$ is a homeomorphism of $K_{* i}^{\prime}$ onto $K_{* i}^{\prime}, f_{t}=g$, and $P_{j} f_{k}=f_{j} P_{j}$ whenever $t \leqq j \leqq k<s+1$. (When $s<\infty, f_{s}$ will be the desired mapping $f_{K^{\prime}, g, K}$. When $s=\infty, f_{K^{\prime}, g, k}$ will 
be defined in terms of the sequence $f_{t}, f_{t+1}, f_{t+2}, \cdots$.) Suppose the mappings $f_{i}$ having the desired properties have been defined for $t \leqq i \leqq m-1$ $(t+1 \leqq m<s+1)$. (For $m=t+1$, this is accomplished by simply taking $f_{t}=g$.) We wish to define $f_{m}$ so that the augmented family $\left\{f_{i} \mid t \leqq i \leqq m\right\}$ still has the properties. For each $y \in K_{* m-1}$, let $l y=\left(l_{m} y\right)^{m}$ and $h y=\left(h_{m} y\right)^{m}$, and similarly with $l^{\prime}$ and $h^{\prime}$ on $K_{* m-1}$. Notice that if $y^{\prime} \in K_{* m-1}^{\prime}$ and $l^{\prime} y^{\prime}=h^{\prime} y^{\prime}$, then with $\eta^{\prime} \in K^{\prime}$ and $y^{\prime}=\eta_{* m-1}^{\prime}$ we have $\eta^{\prime} \in L_{m}^{\prime} \cap H_{m}^{\prime}$, whence, by (2), $\eta^{\prime} \in E_{m-1}^{\prime}$ and thus $y^{\prime} \in F K_{* m-1}^{\prime}$. Since the homeomorphism $f_{m-1}$ must preserve boundary points, $f_{m-1} y^{\prime}=y \in F K_{* m-1}$. But then with $\eta \in K$ and $y=\eta_{* m-1}$ we have $\eta \in E_{m-1}$, whence, by (2), $\eta \in L_{m} \cap H_{m}$ and $l y=h y$. Since the argument is valid also when the roles of $K_{* m-1}^{\prime}$ and $K_{* m-1}$ are reversed, we see that $l y=h y$ iff $l^{\prime} y^{\prime}=h^{\prime} y^{\prime}$ (where $\left.y=f_{m-1} y^{\prime}\right)$. Now for each $y^{\prime} \in K_{* m-1}^{\prime}$ let $f_{m}$ map the segment $\left[\left(y^{\prime}, l^{\prime} y^{\prime}\right)\right.$, $\left.\left(y^{\prime}, h^{\prime} y^{\prime}\right)\right] \subset K_{* m}^{\prime}$ linearly (and preserving order) onto the segment $[(y, l y)$, $(y, h y)] \subset K_{* m}$. From the remarks made above it follows that $f_{m}$ is a biunique mapping of $K_{* m}^{\prime}$ onto $K_{* m}$. The argument of Keller shows that $f_{m}$ is a homeomorphism and that the agumented family has the desired properties. Proceeding by mathematical induction, the family $\left\{f_{i} \mid t \leqq i<s+1\right\}$ is obtained. For $s<\infty, f_{s}$ is the map $f_{K^{\prime}, g, K}$. When $s=\infty$, define $f_{s}$ as follows: $\left(f_{\infty} x^{\prime}\right)_{* n}=f_{n} x_{* n}^{\prime}$ for each $x^{\prime} \in K^{\prime}$ and $n \geqq t$. The argument of Keller shows that $f_{\infty}$ is the desired map $f_{K^{\prime}, g, K}$, so the description of these maps is complete.

When $g$ is defined only on $K_{* 0}^{\prime}=\{\phi\}$, the resulting map $f_{K^{\prime}, o, K}$ will be denoted by $f_{K^{\prime}, K}$.

A necessary lemma is

(4.1) Suppose that $X_{\alpha}$ and $Y_{\alpha}$ are sequences of convex sets in $E^{n}$, converging to $X$ and $Y$ respectively, that $Y$ is a strictly convex body, and $L=\lim \inf \left(X_{\alpha} \cap Y_{\alpha}\right)$ is nonempty. Then $X \cap Y=\lim \left(X_{\alpha} \cap Y_{\alpha}\right)$.

Proof. Using merely the convexity of the $Y_{i}$ 's, we see that $(\dagger) X \cap$ Int $Y$ $\subset L \subset \lim \sup \left(X_{\alpha} \cap Y_{\alpha}\right) \subset X \cap Y$, for two of the inclusions are obvious and we shall show that $X \cap$ Int $Y \subset L$. Consider an arbitrary point $p \in X \cap$ Int $Y$. Since each $Y_{i}$ is convex, there are an integer $N$ and an open set $U \ni p$ such that $U \subset Y_{i}$ whenever $i>N$. (See $[14,(5.2)]$.) Since $p \in \lim X_{\alpha}$, there are a sequence $x_{\alpha} \rightarrow p$ such that always $x_{i} \in X_{i}$ and an integer $M$ such that $x_{i} \in U$ whenever $i>M$. Thus $i>\max (M, N)$ implies $x_{i} \in X_{i} \cap Y_{i}$, whence $p \in L$ and $X \cap$ Int $Y \subset L$.

Now with $L$ nonempty, the conclusion of (4.1) clearly follows from $(\dagger)$ in case $X \cap Y$ contains no more than one point, so we need consider only the case of nondegenerate $X \cap Y$. Then if the conclusion is false, there is a point $x \in X \cap Y \sim L$ and a point $v \in X \cap Y \sim\{u\} . X$ must be convex and $Y$ is strictly convex, so $] u, v[\subset X \cap$ Int $Y$. Since $L$ is closed and $u \notin L$, we see that $X \cap$ Int $Y \nsubseteq L$, which contradicts $(\dagger)$ and completes the proof.

(4.2) Suppose $K^{\prime}, K_{0}, K_{1}, K_{2}, \cdots$ are compact elliptically convex subsets of $\mathfrak{S}_{* m}$, with $0 \leqq m \leqq \infty$, such that $K_{\alpha} \rightarrow K_{0}$. Suppose $x_{\alpha}$ is a sequence of points of $K^{\prime}$ with $x_{\alpha} \rightarrow x_{0}$. Then $f_{K^{\prime}, K_{\alpha}} x_{\alpha} \rightarrow f_{K^{\prime}, K_{0}} x_{0}$. 
Proof. When $m=0$ this is obvious. Suppose it has been proved for $m=n-1$ and consider the case $m=n$. Notice that $K_{\alpha * n-1} \rightarrow K_{0 * n-1}$ and $x_{\alpha * n-1} \rightarrow x_{0 * n-1}$, so from the inductive hypothesis it follows that $f_{K^{\prime}{ }_{* n-1}, K_{\alpha * n-1}} x_{\alpha * n-1}$ $=y_{\alpha} \rightarrow f_{K^{\prime}{ }_{* n-1}, K_{0 * n-1}} x_{0 * n-1}$. Let $\lambda_{\beta}^{\prime}$ resp. $\lambda_{\beta}$ be the line in $\mathfrak{F}_{* n}$ which passes through $x_{\beta * n-1}$ resp. $y_{\beta}$ and is perpendicular to $\mathfrak{S}_{* n-1}$. Then $f_{K^{\prime}, K_{\beta}}$ maps the segment $\sigma_{\beta}^{\prime}=\lambda_{\beta}^{\prime} \cap K^{\prime}$ onto the segment $\sigma_{\beta}=\lambda_{\beta} \cap K_{\beta}$ in a manner which is biunique, linear, and preserves the order of $n$ th-coördinates. From the lemma (4.1) it follows that $\sigma_{\alpha}^{\prime} \rightarrow \sigma_{0}$ and $\sigma_{\alpha} \rightarrow \sigma_{0}$, whence the desired conclusion follows readily for the case $m=n$, and hence, by mathematical induction, for all $m<\infty$. We still must consider the case $m=\infty$.

Suppose there exist sets and points as described in $\mathfrak{S E}_{*_{\infty}}=\mathfrak{S}$, such that $f_{K^{\prime}, K_{\alpha}} x_{\alpha}$ does not approach $y=f_{K^{\prime}, K_{0}} x_{0}$. From compactness considerations it follows that there are a subsequence $(\gamma)$ of $(\alpha)$ and a point $q \in K_{0}$ such that $f_{K^{\prime}, K_{\gamma}} x_{\gamma} \rightarrow q \neq y$. But the finite-dimensional result shows that $q_{* n}=f_{K_{* n}^{\prime}, K_{0 * n}} x_{0 * n}$ $=\left(f_{K^{\prime}, K_{0}} x_{0}\right)_{* n}$ for each $n$, whence $q=y$, a contradiction completing the proof of (4.2).

The remainder of this section will be devoted to establishing the $n$-point homogeneity of the Hilbert parallelotope.

The following result is established by Keller:

(4.3) Suppose $K$ is a bounded convex subset of Hilbert space $\mathfrak{S}$, having $\phi$ as a nonsupport point, and that $\eta \in K$ is such that $K \cap \Re \eta=[-\eta, \eta]$. Then there is a function $\mu$ on $K$ to $\Re$ such that with $k x=(\mu x) x$ for each $x \in K, k$ is a homeomorphism of $K$ onto an elliptically convex set $K^{\prime}$ such that $k \eta=\eta, k(-\eta)=-\eta$, and $\zeta \cdot \eta<\eta \cdot \eta$ whenever $\zeta \in K^{\prime}-\{\eta\}$.

Let $\delta_{\beta} \in H$ be defined by: $\delta_{\beta}^{i}=1$ for $i=\beta$ and $\delta_{\beta}^{i}=0$ for $i \neq \beta$.

(4.4) Suppose $x_{1}, x_{2}, \cdots, x_{j}$ are distinct points of the Hilbert parallelotope $\mathfrak{B}=\prod_{1}^{\infty}[-1 / n, 1 / n]$. Then there is a homeomorphism $h$ of $\mathfrak{B}$ onto an elliptically convex set $Q \subset \mathfrak{S}$ such that for each $i, h x_{i}=\delta_{i}, Q \cap \Re \delta_{i}=\left[-\delta_{i}, \delta_{i}\right]$ and $\left|x^{i}\right|<1$ whenever $x \in Q \sim\left\{\delta_{i},-\delta_{i}\right\}$.

Proof. Let $J=\left\{x_{1}, x_{2}, \cdots, x_{j}\right\}$. We describe first a homeomorphism $f$ of $\mathfrak{B}$ onto $\mathfrak{B}$ such that $f J$ is linearly independent and $\Re y \cap \mathfrak{B}=[-y, y]$ for each $y \in f J$. Let $G=\left\{x \mid x \in J\right.$ and $\left|x^{n}\right|=1 / n$ for some $\left.n\right\}$, and let $S$ be a finite subset of positive integers such that $x \in G$ implies $\left|x^{n}\right|=1 / n$ for some $n \in S$. There is an integer $N$ such that $J_{* N}$ contains $n$ points. Let $M$ be an integer such that $M>j, M>N$, and $\{1, \cdots, M\} \supset S$. It is easy to describe a homeomorphism $w$ of $\mathfrak{B}_{* M}$ onto itself such that $w J_{* M}$ is linearly independent.

Let $\left\{Q_{x} \mid x \in J \sim G\right\}$ be a family of pairwise disjoint infinite sets whose union is $\{i \mid i>M\}$. For $n \in Q_{x}$ let $r_{n}=1 / n-1 / 2 n^{2}$ and let $f_{n}$ be the homeomorphism of $[-1 / n, 1 / n]$ onto itself which maps $\left[-1 / n, x^{n}\right]$ resp. $\left[x^{n}, 1 / n\right]$ linearly onto $\left[-1 / n, r_{n}\right]$ resp. $\left[r_{n}, 1 / n\right]$. Now for each $y \in \mathfrak{B}$, let $f y$ be given by: $(f y)_{* M}=w y_{* M}$ and $(f y)^{n}=f_{n} y^{n}$ for $n>M$. Then $f$ is a homeomorphism of $\mathfrak{B}$ onto itself such that $(f J)_{* M}$, and hence $f J$, is linearly independent. Now if $y \in G$, then $(f y)_{* M}$ is in the boundary of $P_{* M}$. If $y \in J \sim G$, then $(f y)^{n}=r_{n}$ for 
each $n$ in the infinite set $Q_{y}$; for $t>1, t r_{n}=(1 / n) t(1-1 / 2 n)$, which is $>1 / n$ for all sufficiently large $n$. Thus in either case, $\mathfrak{B} \cap \Re f y=[-f y, f y]$, and $f$ has the stated properties.

Since $f J$ is linearly independent, there is a linear homeomorphism $g$ of $\mathfrak{S}$ onto $\mathfrak{S}$ such that $g f x_{i}=\delta_{i}$ for $1 \leqq i \leqq n$. Now let $k_{1}$ be the homeomorphism $k$ obtained in (4.3) with $K=g \Re$ and $\eta=\delta_{1}$. Having defined $k_{i}$ for $1 \leqq i \leqq m-1$ (with $m \leqq n$ ), let $k_{m}$ be the homeomorphism of (4.3) with $K=k_{m-1} k_{m-2} \ldots$ $k_{2} k_{1} g \mathfrak{B}$ and $\eta=k_{m-1} k_{m-2} \cdots k_{2} k_{1} \delta_{m}$. Let $h^{\prime}=k_{m} k_{m-1} \cdots k_{2} k_{1} g f$. Since each map $k_{i}$ is radial from $\phi, h^{\prime}$ has all the desired properties except that $h^{\prime} x_{i}$ may be some positive multiple of $\delta_{i}$ other than $\delta_{i}$ itself. Then obviously there is a linear homeomorphism $u$ such that $h=u h^{\prime}$ is the desired map, completing the proof of (4.4).

(4.5) Theorem. Suppose $K$ is an infinite-dimensional compact convex subset of a normed linear space, $n$ is a positive integer, and that each of the sets $\left\{x_{1}, \cdots, x_{n}\right\}$ and $\left\{y_{1}, \cdots, y_{n}\right\}$ consists of $n$ distinct points of $K$ 〈that the set $\left\{x_{1}, \cdots, x_{n}, y_{1}, \cdots, y_{n}\right\}$ consists of $2 n$ distinct points of $\left.K\right\rangle$. Then there is a homeomorphism $h$ 〈of period 2 〉 of $K$ onto $K$ such that always $h x_{i}=y_{i}$.

Proof. It suffices to consider only the case $K=\mathfrak{B}$, since $K$ is homeomorphic with $\mathfrak{B}$. The first assertion (omitting the words in \langle\rangle ) is merely that $\mathfrak{B}$ is $n$-point homogeneous. Now by (4.4) there is a homeomorphism $h_{x}$ of $\mathfrak{B}$ onto an elliptically convex set $Q_{x} \subset \mathfrak{S}$ such that always $h_{x} x_{i}=\delta_{i}$ and $\delta_{i} \in F Q_{x * i}$, and the same statement is true with $x$ replaced by $y$ throughout. The natural radial map $g$ of $Q_{x * n}$ onto $Q_{\nu * n}$ is a homeomorphism taking always $\delta_{i}$ onto itself, and thus the map $f=f_{Q_{x}, g, Q_{y}}$ is a homeomorphism of $Q_{x}$ onto $Q_{y}$ such that always $f \delta_{i}=\delta_{i}$. Then $h=h_{y}^{-1} f h_{x}$ is a homeomorphism of $\mathfrak{P}$ onto $\mathfrak{B}$ with $h x_{i}=y_{i}$ for each $i$.

To establish the second assertion of (4.5) suppose the points $x_{i}$ and $y_{i}$ are all distinct and let $w$ be a homeomorphism of $\mathfrak{P}$ onto $\mathfrak{P}$ such that always $w x_{i}=\delta_{i}$ and $w y_{i}=-\delta_{i}$. Let $r$ be the reflection map $r x=-x \mid x \in \mathfrak{B}$. Then $h=w^{-1} r w$ is a homeomorphism of period 2 of $\mathfrak{B}$ onto $\mathfrak{B}$, interchanging $x_{i}$ and $y_{i}$ for each $i$.

5. Topological representatives of convex sets. Throughout this section, $C$ will denote a closed convex subset of a normed linear space. We would like to find a simple description of the various topological possibilities for $C$, and are here able to do this for locally compact $C$. Still open is the interesting problem of determining all topological possibilities for $C \subset \mathfrak{S}$, and in particular of deciding whether there are uncountably many such possibilities. (In this connection, see $[13$, p. 31$]$.)

The main theorem of this section ((5.8), also stated in the Introduction) will be based on several lemmas. The first of these can be proved by the argument of (2.2) (iv) (earlier in this paper), the second by that of (III 1.5) in [13]. 
(5.1) Suppose $S$ is a closed subset of a normed linear space and is star-shaped from the point p. Then (a) and (b) below are equivalent and imply (c): (a) every bounded closed subset of $S$ is compact; (b) p has a compact neighborhood in $S$; (c) $S$ is bounded iff it contains no ray emanating from $p$.

(5.2) Suppose $L$ and $L^{\prime}$ are complementary closed linear subspaces of a normed linear space $E, C$ is a closed convex subset of $E, L \subset C$, and $C^{\prime}=C \cap L^{\prime}$. Then $C=L+C^{\prime}$ and hence $C$ is homeomorphic with $L \times C^{\prime}$.

We next recall a notion introduced by Steinitz and employed also by Stoker [19] and the author [13]. For $u \in C, C_{u} \equiv\{u\} \cup\{p \mid \operatorname{Ray}[u, p[\subset C\}$ and is called a characteristic cone of $C$. $C_{u}$ is a closed convex cone with vertex $u$, and if also $v \in C$, then $C_{u}=C_{v}+(u-v)$.

(5.3) If $C$ is a locally compact closed convex subset of a normed linear space, and the characteristic cone of $C$ is a linear manifold, then there are cardinal numbers $m$ and $n$ with $0 \leqq m \leqq \boldsymbol{\aleph}_{0}$ and $0 \leqq n<\boldsymbol{\aleph}_{0}$ such that $C$ is homeomorphic with $\left.[0,1]^{m} \times\right] 0,1{ }^{n}$.

Proof. We may assume $\phi \in C$. Then $C_{\phi}$ is a locally compact linear subspace, hence is finite-dimensional and homeomorphic with $] 0,1\left[{ }^{n}\right.$ for some finite $n$. Being finite-dimensional, $C_{\phi}$ is closed and must admit a closed complementary subspace $L^{\prime}$. With $C^{\prime}=C \cap L^{\prime}$, it follows from (5.1) that $C^{\prime}$ is compact. If $C^{\prime}$ is infinite-dimensional, it is homeomorphic with $[0,1]^{N_{0}}$ by (1.2); if $n$-dimensional, with $[0,1]^{n}$ by $[13$, (III 1.6) $]$. The statement of (5.3) then follows from (5.2).

It remains to consider the case in which $C^{\prime}$ 's characteristic cone is not a linear manifold. We proceed to develop the necessary lemmas.

(5.4) Suppose $g$ is a real-valued continuous function on $[0, \infty[$ such that whenever $0 \leqq t \leqq 1$ and $0 \leqq r<s$ it is true that $g r>g s, r(g r)<s(g s)$, and $(g r)(g s)$ $\leqq[t(g s)+(1-t)(g r)] g(t r+[1-t] s)$. Suppose $E$ is a normed linear space, $K$ is a convex subset of $E$ which contains the neutral element $\phi$, and $T x=(g\|x\|) x$ for each $x \in E$. Then $T K$ is a convex set which is homeomorphic with $K$. If \|\| is strictly convex and $\phi$ is a nonsupport point of $K$, then $T K$ is elliptically convex. If $K$ is closed and contains no ray, then $T K$ is closed in $E$.

Except for the last assertion, this can be proved by an argument very similar to that of $[13,($ IV 1.2) $]$, and the last assertion is not difficult to prove. Observe that with $g r=(1+r)^{-1} \mid r \in[0, \infty[$, the conditions of (5.4) are satisfied and $\|T x\|<1$ for each $x \in E$. Thus

(5.5) If $E$ is a normed linear space and $C$ is a closed convex subset of $E$ which contains no ray, then $C$ is homeomorphic with a bounded closed convex subset of $E$.

(5.6) Suppose $W$ is a normed linear space, $E=\Re \times W, Q=[0, \infty[\times W$, $A=[0, \infty[\times\{\phi\}$ and $J=\{(t, w) \mid t \geqq\|w\|\}$. For each $(t, w) \in Q$, let $T(t, w)$ $=(t+\|w\|, w)$. The $T$ is a homeomorphism of $Q$ onto $J$ such that $T K$ is convex and $T K+A \subset T K$ whenever $K$ is convex and $K+A \subset K \subset Q$.

Proof. It is easy to verify that $T$ is a homeomorphism of $Q$ onto $J$. Now 
consider $K$ as described and arbitrary $(r, x) \in K,(s, y) \in K, \lambda \in[0,1]$, and $(t, \phi) \in A$. We wish to show that $T K$ includes the point $p=\lambda T(r, x)$ $+(1-\lambda) T(s, y)+(t, \phi)$. Now $p=T q$, where $q=(\lambda r+[1-\lambda] s+D, \lambda x+[1-\lambda] y)$ and $D=\lambda\|x\|+(1-\lambda)\|y\|-\|\lambda x+(1-\lambda) y\|+t$. Since $K$ is convex and $D \geqq 0$, $q \in K+A \subset K$ and the proof is complete.

With $\mathfrak{B}$ denoting the Hilbert parallelotope, let $\mathfrak{P}^{\prime}$ be an elliptically convex homeomorph of $\mathfrak{B}$ such that $\mathfrak{B}^{\prime} \subset \mathfrak{C}$ and $\phi$ is a nonsupport point of $\mathfrak{P}^{\prime}$. Let $Z=\left\{(t, t x) \mid t \geqq 0, x \in \mathfrak{B}^{\prime}\right\} \subset \Re \times \mathfrak{S}$. ( $Z$ is homeomorphic with the space obtained from $\mathfrak{B} \times[0,1$ [ by identifying as one point the set $\mathfrak{B} \times\{0\}$.)

(5.7) With $E, Q$, and $A$ as in (5.6), suppose $C$ is an infinite-dimensional locally compact closed convex subset of $E$ such that $C+A \subset C \subset Q$. Then $C$ is homeomorphic with $Z$.

Proof. Let $T$ be as in (5.6) and let $D^{\prime}=T C$. Then $D^{\prime}$ is a closed convex homeomorph of $C$ such that $D^{\prime}+A \subset D^{\prime} \subset J$. Since $D^{\prime}$ is locally compact, and $J \cap\{(a, x) \mid a \leqq b\}$ is bounded for each $b \in \Re$, it follows from (5.1) that there is a point $(s, y) \in D^{\prime}$ such that $(r, z) \in D^{\prime}$ implies $r \geqq s$. Let $D=T\left[D^{\prime}-(s, y)\right]$. Then $D$ is a closed convex homeomorph of $C$ such that $A \subset D \subset J$, and it follows also that $D+A \subset D$. For each $t \geqq 0$, let $D^{t}=\{w \mid w \in w$ and $(t, w) \in D\}$. Each $D^{t}$ is bounded and hence by (5.1) must be compact. Since $D+A \subset D$ and $D$ is star-shaped from $(0, \phi)$, we have $D^{r} \subset D^{s} \subset s r^{-1} D^{r}$ whenever $0 \leqq r \leqq s$. Since $D^{1}$ is separable, there is a sequence $g_{\alpha}$ of continuous linear functionals on $W$ such that $0<\max _{D^{1}}\left|g_{\beta}\right|<2^{-\beta}$, and such that for each pair $x$ and $x^{\prime}$ of distinct points of $D^{1}$ there is an $n$ for which $g_{n} x \neq g_{n} x^{\prime}$. Now for each $(t, w) \in D$ let $S(t, w)=\left(t, y^{1}, y^{2}, \cdots\right)$, where $y^{\beta}=g_{\beta} w$. Since

$$
\max _{D^{t}}\left|g_{\beta}\right| \leqq \max (1, t) 2^{-\beta}
$$

$S$ maps $D$ linearly into $\Re \times \mathfrak{E}=\Re \times\left(l^{2}\right)$. As in (1.1), it can be seen that $S$ is a homeomorphism. Thus $S D$ is a closed convex homeomorph of $C$ such that $A^{\prime} \subset S C \subset Q^{\prime}$, where $A^{\prime}, Q^{\prime}$, and $J^{\prime}$ result from the definitions of (5.6) by taking $W=\mathfrak{S}$. Applying the transformation of (5.6) once more, we obtain a closed convex homeomorph $D^{\prime \prime}$ of $C$ such that $A^{\prime} \subset D^{\prime \prime} \subset J^{\prime}$. Thus it follows that in proving (5.7) we need consider only the case in which $W=\mathfrak{S}$ and $A \subset C \subset J$. We henceforth restrict our attention to this case and may assume further that $C$ is not contained in any hyperplane in $\mathfrak{R} \times \mathfrak{S}$.

By a theorem of Keller [11], each set $C^{t}=\{h \mid h \in \mathfrak{S}$ and $(t, h) \in C\}, t>0$, must include a nonsupport point $p_{t}$. Let $q_{t}=p_{1}$ for $t \geqq 1, q_{t}=\phi$ for $t \leqq 0$, and $q_{t}=\lambda p_{(n+1)^{-1}}+(1-\lambda) p_{n^{-1}}$ for $t=\lambda(n+1)^{-1}+(1-\lambda) n^{-1}(\lambda \in[0,1], n$ a positive integer). Then $q_{t} \mid t \in R$ is continuous and $q_{t}$ is a nonsupport point of $C^{t}$ whenever $t>0$. For each $(t, h) \in E=\Re \times \mathfrak{S}$, let $w(t, h)=\left(t, T\left(h-q_{t}\right)\right)$, where $T$ is as in (5.4), with $g r=(1+r)^{-1}$ and \|\| the usual (strictly convex) norm of $\mathfrak{S}$. Let $K=w C$. Then $w$ is a homeomorphism of $E$ onto $E$ and for each $t>0, K^{t}$ is elliptically convex and $w C^{t}=K^{t}$. We wish to show that $K$ is homeomorphic with $Z$. 
Note that $Z \subset \mathfrak{R} \times \mathfrak{S}$ is defined by the condition $Z^{t}=t \mathfrak{B}^{\prime}$ for $t \geqq 0$ and $Z^{t}=\Lambda$ for $t<0, \mathfrak{B}^{\prime}$ being an elliptically convex homeomorph of $\mathfrak{B}$. Now let $h(0, \phi)$ $=(0, \phi)$, and for $(t, x) \in Z$ with $t>0$ let $h(t, x)=\left(t, f_{\mathfrak{B}^{\prime}, K^{t}}\left(t^{-1} x\right)\right)$. With the aid of (4.2), it can be verified that $h$ is a homeomorphism of $Z$ onto $K$, completing the proof of (5.7).

(5.8) THEOREM. If $C$ is a locally compact closed convex subset of a normed linear space, then there are cardinal numbers $m$ and $n$ with $0 \leqq m \leqq \boldsymbol{\aleph}_{0}$ and $0 \leqq n<\boldsymbol{N}_{0}$ such that $C$ is homeomorphic with either $\left.[0,1]^{m} \times\right] 0,1\left[^{n}\right.$ or $[0,1]^{m}$ $\times[0,1[$. The various possibilities indicated are all topologically distinct.

Proof. We start by proving the second assertion. Let $C^{*}$ denote the onepoint compactification of $C$. That the formally different possibilities listed above are actually topologically different is a consequence of the following (easily verified) facts. (1) If $C$ is homeomorphic with $[0,1]^{m}$, then $C$ is compact and $\operatorname{dim} C=m$. (2) If $C$ is homeomorphic with $[0,1]^{m} \times[0,1[$, then $C$ is noncompact, $\operatorname{dim} C=m+1$, and $C^{*}$ is contractible. (3) If $n>0$ and $C$ is homeomorphic with $\left.[0,1]^{m} \times\right] 0,1\left[{ }^{n}\right.$, then $\operatorname{dim} C=m+n$ and every continuous map of $S^{k}$ into $C^{*}$ is null-homotopic for $k<n$, although there is an essential map of $S^{n}$ into $C^{*}$.

Now the first assertion follows from (5.3) when $C$ 's characteristic cone is a linear manifold and from [13, (III 1.6)] when $C$ is finite-dimensional. Consider the remaining case and assume $\phi \in C$. Since $C_{\phi}$ is not a linear manifold, there is a point $p$ such that $[0, \infty[p \subset C$ but $-p \notin C$, and there is a closed half-space $S \supset C$ such that $-p \notin S$. There is an $m>0$ such that if $S^{\prime}=S+m p$, then $\phi$ is included in the bounding hyperplane $W$ of $S^{\prime}$. Each point $x \in L$ (the normed linear space in which we are working) has a unique expression in the form $w_{x}+t_{x} p$ with $w_{x} \in W$ and $t_{x} \in \Re$. Let $g$ be the map of $L$ onto $E=\Re \times W$ given by $g x=\left(t_{x}, w_{x}\right)$. Then $D=g(C+m p)$ is a closed convex homeomorph of $C$ such that $D+A \subset D \subset Q$, where $A$ and $Q$ are as in (5.7). From (5.7) it follows that $D$ is homeomorphic with $Z$, and also that $[0,1] \aleph_{0} \times[0,1$ [ (equivalently, $\mathfrak{P} \times[0,1[)$ is homeomorphic with $Z$, so the proof is complete.

\section{REFERENCES}

1. Richard Arens, Extension of functions on fully normal spaces, Pacific Journal of Mathematics vol. 2 (1952) pp. 11-22.

2. Stefan Banach, Théorie des opérations linéaires, Warsaw, 1932.

3. N. Bourbaki, Espaces vectoriels topologiques, Actualités Scientifiques et Industrielles, no. 1189, Paris, 1953.

4. C. H. Dowker, An imbedding theorem for paracompact metric spaces, Duke Math. J. vol. 14 (1947) pp. 639-645.

5. J. Dugundji, An extension of Tietze's theorem, Pacific Journal of Mathematics vol. 1 (1951) pp. 353-367.

6. Ralph H. Fox, Extension of homeomorphisms into Euclidean and Hilbert parallelotopes, Duke Math. J. vol. 8 (1941) pp. 452-456.

7. Maurice Fréchet, Les espaces abstraits, Paris, 1928. 
8. F. Hausdorff, Erweiterung einer stetigen Abbildung, Fund. Math. vol. 30 (1938) pp. 4047.

9. Witold Hurewicz and Henry Wallman, Dimension theory, Princeton, 1941.

10. Shizuo Kakutani, Topological properties of the unit sphere of Hilbert space, Proc. Imp. Acad. Tokyo vol. 19 (1943) pp. 269-271.

11. Ott-Heinrich Keller, Die Homoiomorphie der kompakten konvexen Mengen im Hilbertschen Raum, Math. Ann. vol. 105 (1931) pp. 748-758.

12. V. L. Klee, Jr., Convex sets in linear spaces, Duke Math. J. vol. 18 (1951) pp. 443-466.

13. - Convex bodies and periodic homeomorphisms in Hilbert space, Trans. Amer. Math. Soc. vol. 74 (1953) pp. 10-43.

14. - The critical set of a convex body, Amer. J. Math. vol. 75 (1953) pp. 178-188.

15. S. Lefschetz, Topics in topology, Princeton, 1942.

16. G. W. Mackey, On convex topological linear spaces, Trans. Amer. Math. Soc. vol. 60 (1946) pp. 519-537.

17. S. Mazur, Über die kleinsten konvexe Mengen, die eine gegebene kompakte Menge enthalt, Studia Math. vol. 2 (1930) pp. 7-9.

18. W. Sierpinski, Sur les espaces métriques localement séparables, Fund. Math. vol. 21 (1933) pp. 107-113.

19. J. J. Stoker, Unbounded convex point sets, Amer. J. Math. vol. 62 (1940) pp. 165-179.

20. A. H. Stone, Paracompactness and product spaces, Bull. Amer. Math. Soc. vol. 54 (1948) pp. 969-977.

21. A. Tychonoff, Ein Fixpunktsatz, Math. Ann. vol. 111 (1935) pp. 767-776.

22. Gordon Whyburn, Analytic topology, Amer. Math. Soc. Colloquium Publications, vol. 28, New York, 1942.

UNIVERSITY OF WASHINGTON, Seatrle, Wash. 